\title{
Cloning and Identification of Human Syntaxin 5 as a Synaptobrevin/VAMP Binding Protein
}

\author{
Veerasamy Ravichandran and Paul A. Roche* \\ Experimental Immunology Branch, National Cancer Institute, \\ National Institutes of Health, Bethesda, MD
}

Received December 12, 1996; Accepted January 8, 1997

\begin{abstract}
Syntaxins are transmembrane proteins that function in regulating transport vesicle docking and fusion with target membranes in neuronal and nonneuronal cells. Vesicle docking is thought to be regulated in part by the specific interactions of syntaxin with a vesicle-associated membrane protein termed synaptobrevin/VAMP. We have cloned a 1557-bp cDNA that encodes the human syntaxin 5 isoform, using a combination of PCR and colony-screening methods. The deduced 301 amino-acid sequence of human syntaxin 5 shares $96 \%$ identity with rat syntaxin 5 . Like rat syntaxin $1 \mathrm{~A}$, human syntaxin 5 binds to synaptobrevin/VAMP in vitro. The identification of human syntaxin 5 as a synaptobrevin/VAMP-binding protein supports the hypothesis that syntaxin 5 regulates protein transport by binding to vesicle-associated membrane proteins.
\end{abstract}

Index Entries: Syntaxin; synaptobrevin; SNAP-25; vesicular transport.

Docking of synaptic vesicles with the presynaptic plasma membrane is thought to be mediated in part by the binding of vesicle-associated membrane proteins (members of the synaptobrevin/ VAMP family) with the presynaptic plasma membrane proteins syntaxin and SNAP-25. Together, these three proteins form a complex that functions as a receptor for the vesicle/membrane fusion machinery, so that, following vesicle docking, membrane fusion can occur (reviewed in Rothman, 1994). These findings have been extended to cells outside of the nervous system, and it is currently believed that homologous synaptobrevin/VAMP, syntaxin, and SNAP-25-like proteins regulate vesicle-mediated protein transport along the secretory pathway in all cell types.

*Author to whom all correspondence and reprint requests should be addressed. 


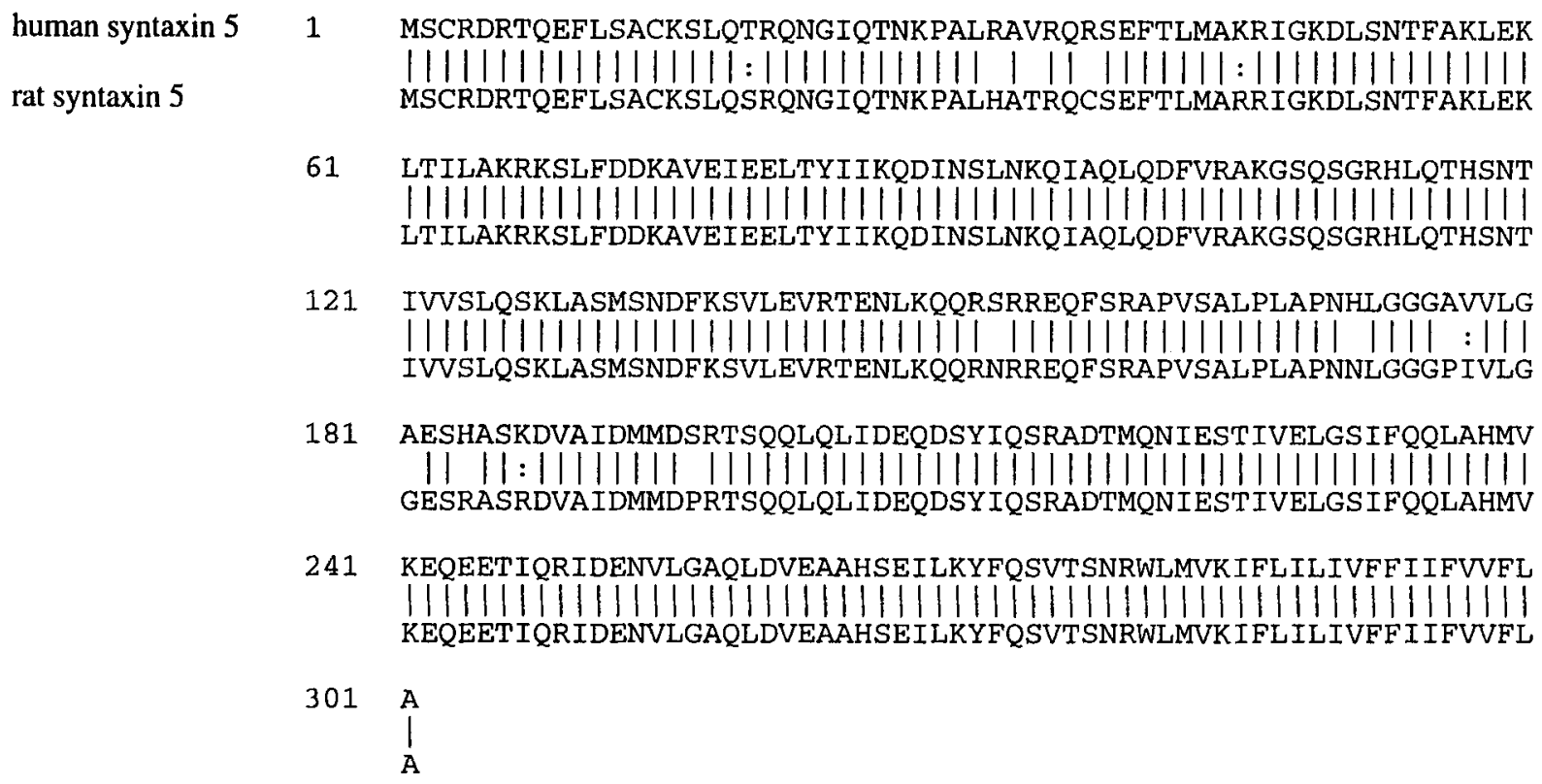

Fig. 1. Comparison of the deduced amino acid sequences of human and rat syntaxin 5 . Numbering begins at the methionine start codon of each syntaxin. Vertical bars represent amino acid identity between the syntaxins; conservative amino acid changes ( $K$ and $R, S$ and $T, V$ and $I)$ are represented by colons. The carboxy-terminal hydrophobic domain of syntaxin is indicated by underscore.

In an attempt to identify syntaxin family members capable of regulating vesicle docking and fusion, Bennett et al. (1993) isolated multiple syntaxins from various rat tissues. One of these syntaxins, syntaxin 5 , is almost $50 \%$ identical to other rat syntaxins and nearly $60 \%$ identical to SED5, a syntaxin-like yeast gene that functions in endoplasmic reticulum-to-Golgi transport in yeast. Syntaxin 5 is localized in the cis-Golgi region of cells (Bennett et al., 1993) and has also been shown to positively regulate endoplasmic reticulum-to-Golgi transport in vitro (Dascher et al., 1994).

Given its localization early in the secretory pathway, we set out to determine if syntaxin 5 is conserved among mammals. Using a human syntaxin 5 probe generated by PCR (corresponding to rat syntaxin 5 amino acids 116-286), we screened 200,000 colonies of an EBV-transformed human lymphocyte cDNA library (Clontech, Palo Alto, CA) to obtain a full-length human syntaxin 5 clone. The filters were hybridized in duplicate, using ${ }^{32} \mathrm{P}$-labeled syntaxin 5 probe in Quick-Hyb
(Stratagene, La Jolla, CA) for $2 \mathrm{~h}$ at $55^{\circ} \mathrm{C}$, and washed three times in $2 \mathrm{X} \mathrm{SSC}, 0.1 \%$ SDS at $55^{\circ} \mathrm{C}$ (Ravichandran et al., 1996). The deduced amino acid sequence of the human syntaxin 5 cDNA (GenBank accession number U26648) shares $96 \%$ identity and $98 \%$ similarity with rat syntaxin 5 (considering conservative amino acid substitutions; Fig. 1). The extensive homology between the human and rat syntaxin 5 isoforms supports the hypothesis that syntaxin 5 is a highly conserved gene necessary for vesicular transport in the early secretory pathway.

Despite sharing approx $50 \%$ amino acid identity with other rat syntaxin family members, direct interactions of syntaxin 5 with other regulators of vesicular transport, namely synaptobrevin/VAMP or SNAP-25, have not been demonstrated. Previous studies have shown that isolated syntaxin proteins, such as syntaxin $1 \mathrm{~A}$, can efficiently bind to glutathione-S-transferase (GST)-fusion proteins of synaptobrevin/VAMP or SNAP-25 in vitro. To determine if syntaxin 5 is also capable of binding to synaptobrevin/VAMP or SNAP-25 directly, we 


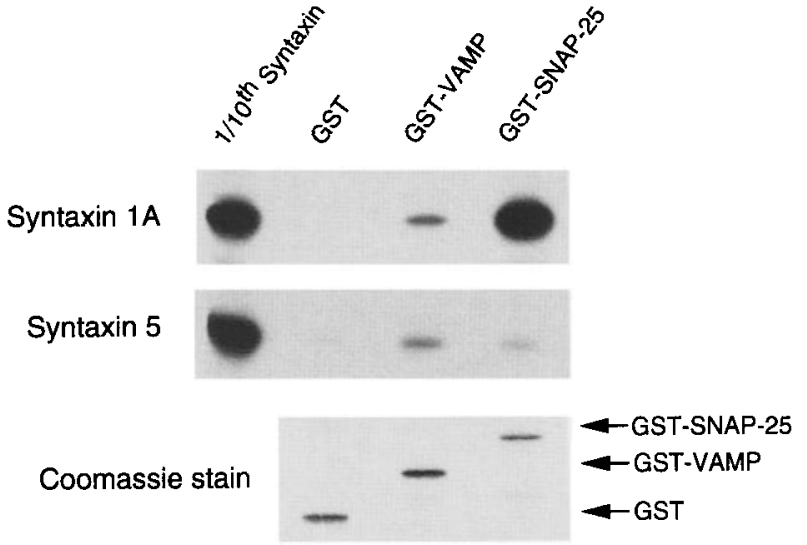

Fig. 2. Binding of Syntaxin $1 \mathrm{~A}$ and Syntaxin 5 to VAMP and SNAP-25 in vitro. Approx $3 \mu \mathrm{g}$ of bacterially expressed GST, GST-VAMP2, or GST-SNAP-25 fusion proteins were incubated with equal amounts of ${ }^{35} \mathrm{~S}$-labeled in vitro-translated rat syntaxin $1 \mathrm{~A}$ or human syntaxin 5 at $4^{\circ} \mathrm{C}$, washed, and analyzed by SDS-PAGE (Ravichandran et al., 1996). The amount of syntaxin bound to the immobilized GST fusion proteins is shown. The gels were stained with Coomassie blue to confirm equal loading of each GST fusion protein (lower panel) and were subjected to autoradiography to detect bound syntaxin (upper panels). One-tenth of the amount of each ${ }^{35} \mathrm{~S}$-labeled syntaxin present in each binding reaction was also run on each gel to help control for translation differences between syntaxin $1 \mathrm{~A}$ and syntaxin 5 . The position of syntaxin $1 \mathrm{~A}$ and syntaxin 5 is indicated.

assayed the ability of in vitro transcribed and translated syntaxin 5 to bind to immobilized GST fusion proteins of rat VAMP2 or human SNAP-25 (Fig. 2). Free syntaxin was separated from bound syntaxin by extensive washing of the glutathione agarose-GST fusion protein beads prior to analysis by SDS-PAGE (Ravichandran et al., 1996). Like syntaxin $1 \mathrm{~A}$, syntaxin 5 did not bind to GST alone, but did bind to GST-VAMP. Syntaxin 5 differed dramatically from syntaxin $1 \mathrm{~A}$ in that it bound to GST-SNAP-25 very poorly. This result is in excellent agreement with a previous study analyzing SNAP-25/syntaxin interactions using the yeast two-hybrid system (Hata and Südhof, 1995).

In this report we have cloned the human homo$\log$ of rat syntaxin 5 . The high degree of identity between rat and human syntaxin 5 is consistent with an essential role for this protein in mammalian cells. In addition, we have demonstrated for the first time that, like other syntaxins, syntaxin 5 is capable of binding to synaptobrevin/VAMP in vitro, suggesting that this protein is a syntaxin, not only based on sequence similarity, but also by function. Unlike other syntaxins, however, syntaxin 5 does not bind efficiently to the target membrane protein SNAP-25 in vitro. It remains to be seen if the poor binding of syntaxin 5 to SNAP25 in some way further enhances the specificity of endoplasmic reticulum-to-Golgi vesicle-mediated protein transport in mammalian cells.

\section{Acknowledgments}

We thank Richard Scheller for the generous gift of the GST-rat VAMP2 fusion protein expression vector. We also thank David Winkler for assistance in automated DNA sequence analysis, Anita Valdez for GST fusion protein preparation, and Dinah Singer and Pierre Henkart for critical reading of this manuscript.

\section{References}

Bennett M. K., Garcia-Arrarás J. E., Elferink L. A., Peterson K., Fleming A. M., Hazuka C. D., and Scheller R. H. (1993) The syntaxin family of vesicular transport receptors. Cell 74, 863-873.

Dascher C., Matteson J., and Balch W. E. (1994) Syntaxin 5 regulates endoplasmic reticulum to Golgi transport. J. Biol. Chem. 269, 29,363-29,366.

Hata Y. and Südhof T. C. (1995) A novel ubiquitous form of Munc-18 interacts with multiple syntaxins. J. Biol. Chem. 270, 13,022-13,028.

Ravichandran V., Chawla A., and Roche P. A. (1996) Identification of a novel syntaxin- and synaptobrevin/VAMP binding protein, SNAP-23, expressed in non-neuronal tissues. J. Biol. Chem. 271, 13,300-13,303.

Rothman J. E. (1994) Mechanisms of intracellular protein transport. Nature 372, 55-63. 